TRANSACTIONS OF THE

AMERICAN MATHEMATICAL SOCIETY

Volume 359, Number 1, January 2007, Pages 165-183

S $0002-9947(06) 03881-5$

Article electronically published on August 16, 2006

\title{
EFFECTIVE INVARIANTS OF BRAID MONODROMY
}

\author{
ENRIQUE ARTAL BARTOLO, JORGE CARMONA RUBER, \\ AND JOSÉ IGNACIO COGOLLUDO AGUSTÍN
}

\begin{abstract}
In this paper we construct new invariants of algebraic curves based on (not necessarily generic) braid monodromies. Such invariants are effective in the sense that their computation allows for the study of Zariski pairs of plane curves. Moreover, the Zariski pairs found in this work correspond to curves having conjugate equations in a number field, and hence are not distinguishable by means of computing algebraic coverings. We prove that the embeddings of the curves in the plane are not homeomorphic. We also apply these results to the classification problem of elliptic surfaces.
\end{abstract}

Our purpose in this paper is the construction of new and effective topological invariants for algebraic curves. These invariants will be derived from the braid monodromy of a triple $(\mathcal{C}, L, P)$ introduced in [3], where $\mathcal{C} \subset \mathbb{P}^{2}$ is an algebraic curve, $L \not \subset \mathcal{C}$ is a line and $P \in L$. This invariant is derived from the classical braid monodromy; see references in 3 .

Several topological invariants of plane algebraic curves, obtained from the fundamental group of the complement, have been found to be effective, such as the Alexander polynomial, the Alexander module or the sequence of characteristic varieties [13]. A second group of effective invariants, described by A. Libgober in [12], depend on polynomial representations of the braid group and are invariants of the conjugation class of the monodromy group.

In this paper, braid monodromy of a triple is represented by an element of $\mathbb{B}_{d}^{r}$ for suitable $r$ and $d$. The set of elements in $\mathbb{B}_{d}^{r}$ representing a braid monodromy coincides with the orbit of a natural action of $\mathbb{B}_{d} \times \mathbb{B}_{r}$ on $\mathbb{B}_{d}^{r}$. Since these orbits are infinite, it is not easy to decide whether or not two elements of $\mathbb{B}_{d}^{r}$ are in the same orbit.

Using finite representations of braid groups we obtain new effective invariants for braid monodromies. Effectiveness is obtained by means of the free software GAP4 [10. We combine these invariants with our main theorem in 3 , which relates braid monodromy to topology.

Braid monodromy may also be useful in the study of the moduli space of projective curves with prescribed degree and topological types of curve singularities. Let $d$ be a positive integer and let $T_{1}, \ldots, T_{r}$ be topological types of curve singularities. Let us denote by $\Sigma\left(T_{1}, \ldots, T_{r} ; d\right)$ the space of all projective plane curves of degree $d$ with exactly $r$ singular points of type $T_{1}, \ldots, T_{r}$. Let $\mathbf{M}\left(T_{1}, \ldots, T_{r} ; d\right)$ be the

Received by the editors January 26, 2004 and, in revised form, October 13, 2004.

2000 Mathematics Subject Classification. Primary 14D05, 14H30, 14H50, 68W30.

Key words and phrases. Braid monodromy, plane curve, group representations.

The first and third authors were partially supported by MTM2004-08080-C02-02. The second author was partially supported by MTM2004-08080-C02-01.

(c)2006 American Mathematical Society Reverts to public domain 28 years from publication 
quotient of $\Sigma\left(T_{1}, \ldots, T_{r} ; d\right)$ by the action of the projective group. Note that braid monodromy is an invariant of each connected component of these moduli spaces.

In this work we also address the problem of the topological type of conjugate varieties. Let $(V, W)$ be a pair of projective varieties, $W \subset V$ ( $W$ may be empty), with defining equations in a number field $K$. For each embedding $j$ of $K \subset \mathbb{C}$ (or for each Galois action of the normal closure of $K / \mathbb{Q}$, if we consider $K$ already embedded in $\mathbb{C}$ ), one obtains a pair $\left(V_{j}, W_{j}\right)$ of complex projective varieties, which essentially shares all the algebraic properties of $(V, W)$.

It is well known that a great number of topological properties of $\left(V_{j}, W_{j}\right)$ are of algebraic nature and they depend only on the pair $(V, W)$. Nevertheless, the works of J.P. Serre [16] and H. Abelson [1] show that it is possible to find examples of non-homeomorphic conjugate varieties, i.e., different embeddings of $K$ provide different topological pairs.

J.P. Serre distinguishes the complex varieties by means of the fundamental group (although the associated algebraic fundamental groups must be isomorphic). Abelson's examples have the same fundamental groups but differ on the cup product in cohomology. Serre's examples are surfaces, whereas Abelson's are higher dimension varieties. Applying generic projection and Chisini's conjecture, Serre's result implies that there are conjugate projective plane curves which do not have the same embedded topological type in the projective plane (probably large degree curves). V. Kharlamov and V. Kulikov [1] have recently proved the existence of non-isotopic pairs of (complex) conjugate algebraic curves. Note that complex conjugate curves are homeomorphically embedded, but the homeomorphism does not respect curve orientations. In addition, they prove that their examples have different generic braid monodromies; the smallest degree of their examples is 825 . In this work we provide examples of the same nature using curves of smaller degree.

According to A.I. Degtyarev's work [8], no examples of non-homeomorphically embedded conjugate curves can exist up to degree 5. In 2], several examples were proposed as candidates. For instance, it was proved that $\mathbf{M}\left(\mathbb{A}_{19} ; 6\right)$ consists of two zero-dimensional connected components. It was also found that a representative of one component, defined by a polynomial in $\mathbb{Q}(\sqrt{5})[x, y, z]$ had, as its conjugate equation, a representative of the other component. This situation will be described by simply saying that $\mathbf{M}$ consists of two points conjugated in $\mathbb{Q}(\sqrt{5})$. In other cases, such as $\mathbf{M}\left(\mathbb{A}_{18}, \mathbb{A}_{1} ; 6\right)$, there are three elements conjugated in a degree 3 extension of $\mathbb{Q}$. In $\left[4\right.$, the family $\mathbf{M}\left(\mathbb{A}_{9}, \mathbb{A}_{9}, \mathbb{A}_{1} ; 6\right)$ was studied. It is easily seen that any curve in $\mathbf{M}\left(\mathbb{A}_{9}, \mathbb{A}_{9}, \mathbb{A}_{1} ; 6\right)$ is the union of a quintic curve with two singular points (one $\mathbb{A}_{9}$ and one $\mathbb{A}_{1}$ ) and a line which intersects the quintic at a smooth point with intersection multiplicity 5 (providing the second $\mathbb{A}_{9}$-point). Following the ideas used in [4] it can be proved that $\mathbf{M}\left(\mathbb{A}_{9}, \mathbb{A}_{9}, \mathbb{A}_{1} ; 6\right)$ has three zero-dimensional connected components. The curves in one component can be distinguished from those in the remaining components using the following algebraic condition: the line joining the $\mathbb{A}_{9}$-points is tangent to the curve. The remaining components have conjugate equations in $\mathbb{Q}(\sqrt{5})$. As mentioned in $[4$, the curves satisfying the geometrical property are not topologically equivalent to those in the other components. This can be done by studying algebraically all possible coverings corresponding to the dihedral group of 10 elements or by calculating their first characteristic varieties.

In this work we will mainly concentrate on the study of the family $\mathbf{M}:=$ $\mathbf{M}\left(\mathbb{E}_{6}, \mathbb{A}_{7}, \mathbb{A}_{3}, \mathbb{A}_{2}, \mathbb{A}_{1} ; 6\right)$. It is easily seen that any curve in $\mathbf{M}$ is the union of a 
quintic curve with three singular points (of types $\mathbb{E}_{6}, \mathbb{A}_{3}$ and $\mathbb{A}_{2}$ ) and a line intersecting the quintic at two smooth points with intersection multiplicities 4 and 1.

Theorem 1. The space $\mathbf{M}$ has exactly two points $\mathbf{M}_{1}$ and $\mathbf{M}_{2}$. There exist representatives $\mathcal{C}_{i} \in \mathbf{M}_{i}, i=1,2$, having conjugate equations with coefficients in $\mathbb{Q}(\sqrt{2})$.

For the sake of simplicity we will not provide a detailed proof of this result. Basically, it is a consequence of applying a standard Cremona transformation, incorporating the ideas used in [4] to the family $\mathbf{M}\left(\mathbb{A}_{15}, \mathbb{A}_{3}, \mathbb{A}_{1} ; 6\right)$, and performing very straightforward calculations. In this paper we also compute the invariants that are needed to deduce that the fundamental groups of the complements are both isomorphic to $\mathbb{Z} \times S L(2, \mathbb{Z} / 7 \mathbb{Z})$.

For any $\mathcal{C} \in \mathbf{M}$, one can construct a curve $\mathcal{C}^{\varphi}$ associated to the triple $(\mathcal{C}, L, P)$ where $P$ is the $\mathbb{E}_{6}$-point and $L$ is the tangent line to $\mathcal{C}$ at $P$ as follows. Let us consider the pencil of lines through $P$. There are exactly five non-generic lines in this pencil different from $L$, namely: the lines joining $P$ to each one of the other four singular points of $\mathcal{C}$ and an ordinary tangent line. Thus, $\mathcal{C}^{\varphi} \cup L$ is the union of a quintic and seven lines. Note that if $\mathcal{C}_{i} \in \mathbf{M}_{i}, i=1,2$, are conjugate curves, this is also the case for $\mathcal{C}_{i}^{\varphi} \cup L$. We will prove the following.

Theorem 2. Let us consider two conjugate curves $\mathcal{C}_{i} \in \mathbf{M}_{i}, i=1,2$. Then, $\mathcal{C}_{1}^{\varphi} \cup L$ and $\mathcal{C}_{2}^{\varphi} \cup L$ are two conjugate curves such that the pairs $\left(\mathbb{P}^{2}, \mathcal{C}_{1}^{\varphi} \cup L\right)$ and $\left(\mathbb{P}^{2}, \mathcal{C}_{2}^{\varphi} \cup L\right)$ are not homeomorphic.

In order to prove this theorem, braid monodromies for the triples $\left(\mathcal{C}_{i}, L, P\right)$, $i=1,2$, are constructed. As in the examples given by Serre and Kharlamov and Kulikov, the braid monodromies are different. Finally, the main theorem in [3] (see also Theorem 2.8) is applied.

These invariants can also yield information in the context of topological invariants of elliptic fibrations. In this paper, an example of two extremal elliptic fibrations with the same combinatorics of special fibers but different homological monodromy is provided.

The following is a general outline of the contents of this paper. In $\S 1$, notations and definitions are set. The definition of braid monodromy of a triple in terms of $r$-tuples of braids is recalled in $\S 2$, followed by the description of a way to produce effective invariants for braid monodromies. Next, in $\S 3$, braid monodromies for representatives in $\mathbf{M}_{1}$ and $\mathbf{M}_{2}$ are calculated and used to prove our main result, Theorem 22 Some other examples are outlined in $\S 4$. Finally, in $\S 5$, an application to the study of elliptic fibrations is presented.

\section{Settings And Definitions}

Let $G$ be a group and $a, b \in G$. We use the following notation: $a^{b}:=b^{-1} a b$, $[a, b]:=a^{-1} b^{-1} a b$ and $b * a:=b a b^{-1}$.

We also denote by $\mathbb{F}_{n}$ the free group on $n$ generators, say $x_{1}, \ldots, x_{n}$, and by $\mathbb{B}_{n}$ the braid group on $n$ strings given by the following presentation:

$$
\left\langle\sigma_{1}, \ldots, \sigma_{n-1}:\left[\sigma_{i}, \sigma_{j}\right]=1,|i-j| \geq 2, \sigma_{i} \sigma_{i+1} \sigma_{i}=\sigma_{i+1} \sigma_{i} \sigma_{i+1}, i=1, \ldots, n-2\right\rangle .
$$

We are going to recall some properties of braid groups (see J. Birman's book $[\underline{5}$ ] for a general reference). Let $V$ be the space of monic polynomials in $\mathbb{C}[t]$ of degree $n\left(V\right.$ and $\mathbb{C}^{n}$ are naturally isomorphic as affine spaces). Let $D$ be the discriminant space of $V$, i.e., the set of elements in $V$ with multiple roots. The space $D$ is an 
algebraic hypersurface of $V$. The space $X:=V \backslash D$ can be naturally identified with the space of subsets of $\mathbb{C}$ having exactly $n$ elements. We will use the following notation:

$$
p(t):=\prod_{j=1}^{n}\left(t-t_{j}\right) \equiv\left\{t_{1}, \ldots, t_{n}\right\}=: \mathbf{y} \text { and } \mathbb{B}_{\mathbf{y}}:=\pi_{1}(X ; \mathbf{y}) .
$$

Let $\mathbf{y}:=\left\{t_{1}, \ldots, t_{n}\right\} \in X$; we identify an element in $\mathbb{B}_{\mathbf{y}}$ with the homotopy class, relative to $\{0,1\}$, of sets of paths $\gamma:=\left\{\gamma_{1}, \ldots, \gamma_{n}\right\}, \gamma_{i}:[0,1] \rightarrow \mathbb{C}$ such that $\gamma(0)=\gamma(1)=\mathbf{y}$ and $\forall t \in[0,1], \gamma(t) \in X$. The elements of $\mathbb{B}_{\mathbf{y}}$ are called braids based at $\mathbf{y}$ and are represented as a set of non-intersecting paths in $\mathbb{C} \times[0,1]$, as usual. Products are also defined in the standard way.

Let $\mathbf{y}:=\left\{t_{1}, \ldots, t_{n}\right\} \in X$. Let us also consider a big enough geometric closed disk $\Delta$ such that $\mathbf{y}$ is contained in the interior of $\Delta$ and let $\star$ be a point on $\partial \Delta$.

Notation 1.1. We will denote by $\gamma_{\infty} \in \pi_{1}(\mathbb{C} \backslash \mathbf{y} ; \star)$ the homotopy class of the loop based at $\star$ which surrounds the circle $\partial \Delta$ counterclockwise.

Recall that $\pi_{1}(\mathbb{C} \backslash \mathbf{y} ; \star)$ is isomorphic to $\mathbb{F}_{n}$ and $\mathbb{B}_{\mathbf{y}}$ is isomorphic to $\mathbb{B}_{n}$.

Example 1.2. Let us consider the following:

- $\mathbf{y}^{0}=\{-1, \ldots,-n\}$,

- $\Delta=\{t \in \mathbb{C}|| t \mid \leq R\}, R \gg 0$,

• $\star=R$.
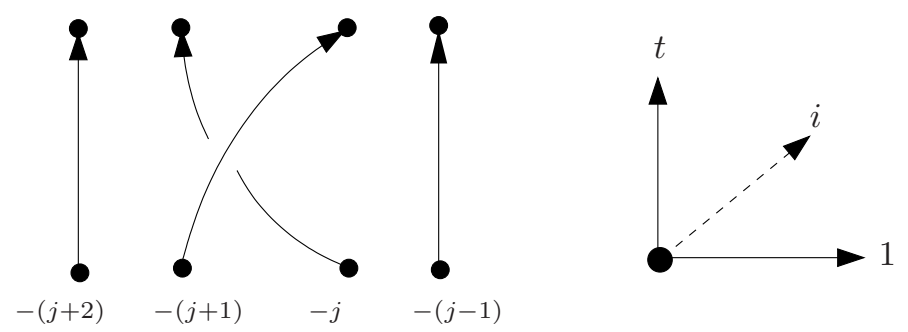

Figure 1. Standard braid $\sigma_{j}$

Let us consider the group $\mathbb{B}_{\mathbf{y}^{0}}$. For $j=1, \ldots, n-1$, the braid $\sigma_{j}$ is defined as a set $\left\{\gamma_{1}^{(j)}, \ldots, \gamma_{n}^{(j)}\right\}$ of paths as in Figure 1, braids are upward and the visible string in each crossing has a smaller imaginary part. It is well known that $\sigma_{1}, \ldots, \sigma_{n-1}$ generate $\mathbb{B}_{\mathbf{y}^{0}}$, providing a geometrical way to identify $\mathbb{B}_{\mathbf{y}^{0}}$ and $\mathbb{B}_{n}$.

Remark 1.3. From now on the abstract group $\mathbb{B}_{n}$ is identified with $\mathbb{B}_{\mathbf{y}^{0}}$.

Based on this example one can describe isomorphisms from $\mathbb{B}_{\mathbf{y}}$ to $\mathbb{B}_{n}$ for any $\mathbf{y} \in X$. This can be achieved by taking into account the fundamental groupoid of $X$, consisting of braids with different ends, i.e., homotopy classes (with fixed ends) of paths in $X$. The set of braids starting at $\mathbf{y}^{1}$ and ending at $\mathbf{y}^{2}$ is denoted by $\mathbb{B}\left(\mathbf{y}^{1}, \mathbf{y}^{2}\right)$. Given a braid $\tau \in \mathbb{B}\left(\mathbf{y}^{1}, \mathbf{y}^{2}\right)$ there is a natural isomorphism $\mathbb{I}_{\tau}: \mathbb{B}_{\mathbf{y}^{1}} \rightarrow$ $\mathbb{B}_{\mathbf{y}^{2}}$ given by $\mathbb{I}_{\tau}(\sigma):=\tau^{-1} \cdot \sigma \cdot \tau$. If $\mathbf{y}^{1}=\mathbf{y}^{2}=\mathbf{y}$, then $\tau \in \mathbb{B}_{\mathbf{y}}, \mathbb{I}_{\tau}$ is the inner automorphism by $\tau$.

We recall the definition of meridians. 
Definition 1.4. Let $Z$ be a connected projective manifold and let $H$ be a hypersurface of $Z$. Let $\star \in Z \backslash H$ and let $K$ be an irreducible component of $H$. A homotopy class $\gamma \in \pi_{1}(Z \backslash H ; \star)$ is called a meridian about $K$ with respect to $H$ if $\gamma$ has a representative $\delta$ satisfying the following properties:

(a) There is a smooth complex analytic disk $\Delta \subset Z$ transverse to $H$ such that $\Delta \cap H=\left\{\star^{\prime}\right\} \subset K$ (transversality implies that $\star^{\prime}$ is a smooth point of $H$ ).

(b) There is a path $\alpha$ in $Z \backslash H$ from $\star$ to $\star^{\prime \prime} \in \partial \Delta$.

(c) $\delta=\alpha \cdot \beta \cdot \alpha^{-1}$, where $\beta$ is the closed path obtained by traveling from $\star^{\prime \prime}$ along $\partial \Delta$ in the positive direction.

It is well known that any two meridians about $K$ with respect to $H$ are conjugate in $\pi_{1}(Z \backslash H ; \star)$. Moreover, any conjugate to a meridian is also a meridian.

Definition 1.5. Let us consider y a point of $X, \Delta \subset \mathbb{C}$ an oriented geometric closed disk containing $\mathbf{y}$ in its interior, and $\star$ a point in $\partial \Delta$. Also, let $\gamma_{\infty} \in \pi_{1}(\mathbb{C} \backslash \mathbf{y} ; \star)$ be as in Notation 1.1. A geometric basis of the free group $\pi_{1}(\mathbb{C} \backslash \mathbf{y} ; \star)$ (with respect to $\Delta)$ is an ordered basis $\mu_{1}, \ldots, \mu_{n}$ such that:

(1) $\mu_{1}, \ldots, \mu_{n}$ are meridians of the points in $\mathbf{y}$;

(2) $\mu_{n} \cdot \ldots \cdot \mu_{1}=\gamma_{\infty}$.

Remark 1.6. The loop $\gamma_{\infty}$ is the inverse of a very special meridian about the point at infinity of $\mathbb{C}$. If we choose the point $\star$ anywhere in $\mathbb{C} \backslash \mathbf{y}$, there is no such special meridian. If $\mu_{1}, \ldots, \mu_{n}$ is a basis of $\pi_{1}(\mathbb{C} \backslash \mathbf{y} ; \star)$ such that $\mu_{n} \cdot \ldots \cdot \mu_{1}$ is the inverse of a meridian of the point at infinity, we will say that $\mu_{1}, \ldots, \mu_{n}$ is a pseudogeometric basis.

Our next goal is to construct canonical isomorphisms between $\mathbb{B}_{\mathbf{y}}$ and $\mathbb{B}_{n}$ for any $\mathbf{y} \in X$. This can be done by fixing a special braid $\tau \in \mathbb{B}\left(\mathbf{y}^{0}, \mathbf{y}\right)$.

Construction 1.7. We will start by constructing lexicographic bases and braids. Let us fix $\mathbf{y} \in X$ and let us consider $\mathbf{y}^{0}$ as above. Let $\Delta$ be a geometric disk (centered at 0 ) containing $\mathbf{y} \cup \mathbf{y}^{0}$ and choose $\star$ as the only positive real number in $\partial \Delta$.

One can order the points $y_{1}, \ldots, y_{n} \in \mathbf{y}$ such that $\Re y_{1} \geq \cdots \geq \Re y_{n}$ and if $\Re y_{j}=\Re y_{j+1}$, then $\Im y_{j}>\Im y_{j+1}$. This ordering is called the lexicographic ordering of $\mathbf{y}$.

We define the lexicographic braid $\tau_{\mathbf{y}}$ associated with $\mathbf{y}$ as the set of paths $\left\{\gamma_{1}, \ldots, \gamma_{n}\right\}$ where $\gamma_{j}(t):=(t-1) j+t y_{j}, j=1, \ldots, n, t \in[0,1]$. Since these paths preserve the lexicographic ordering, they produce a braid. The corresponding isomorphism $\mathbb{I}_{\mathbf{y}}:=\mathbb{I}_{\tau_{\mathbf{y}}}: \mathbb{B}_{n} \rightarrow \mathbb{B}_{\mathbf{y}}$ will be called lexicographic isomorphism from $\mathbb{B}_{n}$ to $\mathbb{B}_{\mathbf{y}}$.

Remark 1.8. Let us consider $\mathbf{y}^{1}, \mathbf{y}^{2} \in X$. Lexicographic braids allow us to consider canonical bijections of $\mathbb{B}\left(\mathbf{y}^{1}, \mathbf{y}^{2}\right)$ where $\mathbb{B}_{n}=\mathbb{B}_{\mathbf{y}^{0}}$ :

$$
\begin{aligned}
\mathbb{B}\left(\mathbf{y}^{1}, \mathbf{y}^{2}\right) & \rightarrow \mathbb{B}_{n} \\
\sigma & \mapsto \tau_{\mathbf{y}^{1}} \cdot \sigma \cdot \tau_{\mathbf{y}^{2}}^{-1} .
\end{aligned}
$$

Let us say we have a braid on $\mathbb{C}$; its graph is a set $E$ of $n$ non-intersecting paths in $\mathbb{C} \times[0,1]$. We explain how to obtain a generic projection onto $\mathbb{R} \times[0,1]$ which by means of lexicographic orderings can be written as a braid in $\mathbb{B}_{n}$. 
- Take the projection $\Re: \mathbb{C} \times[0,1] \rightarrow \mathbb{R} \times[0,1]$ given by the real part on the first coordinate and the identity on the second one. Let us consider its restriction $\Re_{E}$.

- A ramification point of the projection $\Re_{E}$ is a point $p \in \Re(E)$ such that $\# \Re_{E}^{-1}(p)>1$. A ramification point is said to be generic if $p \in \Re(E)$ is a real ordinary double point. At these points, we replace the real node by an overcrossing where the upper branch is the one with the smallest imaginary part.

- At non-generic ramification points (e.g, produced by a pair of conjugate arcs), we slightly perturb the projection in order to have greater imaginary parts to the right and smaller imaginary parts to the left (see [4]). For example, let us suppose that we have two conjugate $\operatorname{arcs} t \mapsto \gamma(t) \pm i$, $\gamma(t) \in \mathbb{R}$. Their perturbed projections will be $\gamma(t)+i \mapsto \gamma(t)+\varepsilon$ and $\gamma(t)-i \mapsto \gamma(t)-\varepsilon$ where $0<\varepsilon \ll 1$.

\section{EFFECTIVE INVARIANTS OF BRAID MONODROMY}

In this section, braid monodromy is described and effective invariants for this class are defined. The definitions have already been introduced in [3, but for the sake of completeness we also present them here. Let $(\mathcal{C}, L, P)$ be a triple where $\mathcal{C} \subset \mathbb{P}^{2}$ is a curve, $L \subset \mathbb{P}^{2}$ is a line not contained in $\mathcal{C}$ and $P \in L$. For convenience we fix homogeneous coordinates $[x: y: z]$ such that $P:=[0: 1: 0], L:=\{z=0\}$ and $\mathcal{C}:=\{f(x, y, z)=0\}$. We identify $\mathbb{C}^{2}=\mathbb{P}^{2} \backslash L$ with affine coordinates $(x, y)$ and we set $\mathcal{C}^{\text {aff }}:=\mathcal{C} \cap \mathbb{C}^{2}$. The pencil $\mathcal{H}_{P}$ of lines through $P$ is given by the vertical lines in the projection $\pi: \mathbb{C}^{2} \rightarrow \mathbb{C}, \pi(x, y):=x$. As a general notation for a curve $\mathcal{C} \subset \mathbb{P}^{2}$ we will use $X_{\mathcal{C}}:=\mathbb{C}^{2} \backslash \mathcal{C}^{\text {aff }}=\mathbb{P}^{2} \backslash(\mathcal{C} \cup L)$.

Definition 2.1. A triple $(\mathcal{C}, L, P)$ is said to be horizontal if $\pi_{\mid \mathcal{C}^{\text {aff }}}: \mathcal{C}^{\text {aff }} \rightarrow \mathbb{C}$ is a proper map. The degree of $(\mathcal{C}, L, P)$ is the degree of $\pi_{\mid \mathcal{C}^{\text {aff }}}$, i.e., the number of preimages of a regular value.

Let $(\mathcal{C}, L, P)$ be a horizontal triple of degree $d$. Note that we can assume that the equation $f(x, y, 1)=0$ for $\mathcal{C}^{\text {aff }}$ is given by

$$
f(x, y, 1)=y^{d}+f_{d-1}(x) y^{d-1}+\cdots+f_{1}(x) y+f_{0}(x),
$$

where $f_{j}(x) \in \mathbb{C}[x], j=0,1, \ldots, d-1$. The condition of being horizontal is equivalent to the non-existence of vertical asymptotes (vertical lines included). Note that the set of critical values is the set of zeros of the discriminant of $f$ with respect to $y$, which is a polynomial $\mathfrak{D}(x) \in \mathbb{C}[x]$. Denote this set of zeros by $\mathscr{D}:=\left\{x_{1}, \ldots, x_{r}\right\}$. Therefore, the special fibers of $\pi$ are exactly the vertical lines $L_{i}:=\pi^{-1}\left(x_{i}\right)$ of equation $x=x_{i}, i=1, \ldots, r$. The non-generic elements of $\mathcal{H}_{P}$ are the projective closures $\bar{L}_{i}=L_{i} \cup\{P\}$ of $L_{i}, i=1, \ldots, r$. We will denote by $\mathscr{L}$ the union of these $r$ projective lines.

Definition 2.2. Let $(\mathcal{C}, L, P)$ be a horizontal triple. Using the above notation, the fibered curve associated with the triple is the curve $\mathcal{C}^{\varphi}:=\mathcal{C} \cup \mathscr{L}$.

Let $\breve{\mathcal{C}}:=\pi_{\mid \mathcal{C}^{\text {aff }}}^{-1}(\mathbb{C} \backslash \mathscr{D})$. Since $\pi_{\mid \mathcal{C}^{\text {aff }}}$ is proper, $\tilde{\pi}: \check{\mathcal{C}} \rightarrow \mathbb{C} \backslash \mathscr{D}$ is a covering map, where $\check{\mathcal{C}}$ might not be connected. The mapping $\pi^{\varphi}: X_{\mathcal{C}^{\varphi}} \rightarrow \mathbb{C} \backslash \mathscr{D}$ is a locally trivial fibration whose fiber is diffeomorphic to $\mathbb{C} \backslash\{1, \ldots, d\}$. The polynomial $f$ induces an algebraic mapping $\tilde{f}: \mathbb{C} \backslash \mathscr{D} \rightarrow X$ defined as $\tilde{f}\left(x_{0}\right):=\left\{x=x_{0}\right\} \cap \mathcal{C}^{\text {aff }}$, 
or, equivalently, as the polynomial $f\left(x_{0}, t\right) \in \mathbb{C}[t]$. Let us fix $\star \in \mathbb{C} \backslash \mathscr{D}$, a complex regular value on the boundary of a geometric disk $\Delta$ containing $\mathscr{D}$ in its interior. Let us denote by $\mathbf{y}^{\star}$ the set of roots of the polynomial $f(\star, t)$.

Let us consider the homomorphism $\nabla_{\star}: \pi_{1}(\mathbb{C} \backslash \mathscr{D} ; \star) \rightarrow \mathbb{B}_{\mathbf{y}^{\star}}$ induced by $\tilde{f}$. This homomorphism may be represented by an $r$-tuple of braids as follows. Let us fix a geometric basis with respect to $\Delta$, say $\mu_{1}, \ldots, \mu_{r}$. Let us choose a braid $\beta \in \mathbb{B}\left(\mathbf{y}^{\star}, \mathbf{y}^{0}\right)$ and consider the isomorphism $\mathbb{I}_{\beta}: \mathbb{B}_{\mathbf{y}^{\star}} \rightarrow \mathbb{B}_{n}$. These considerations allow us to represent $\nabla_{\star}$ by an element $\left(\tau_{1}, \ldots, \tau_{r}\right) \in \mathbb{B}_{d}^{r}$ such that

$$
\tau_{j}:=\mathbb{I}_{\beta}\left(\nabla_{\star}\left(\mu_{j}\right)\right), \quad \forall j=1, \ldots, r .
$$

In order to construct $\left(\tau_{1}, \ldots, \tau_{r}\right)$ we have chosen

- a geometric disk $\Delta$ containing $\left\{x_{1}, \ldots, x_{r}\right\}$ in its interior,

- an element $\star \in \partial \Delta$ such that $\mathbf{y}^{\star}$ is the set of roots of the polynomial $\tilde{f}(\star) \in \mathbb{C}[t]$,

- a geometric basis $\mu_{1}, \ldots, \mu_{r}$ of $\pi_{1}(\mathbb{C} \backslash \mathscr{D} ; \star)$ and

- a braid $\beta \in \mathbb{B}\left(\mathbf{y}^{\star}, \mathbf{y}^{0}\right)$.

Remark 2.3. Let us consider two natural right actions on $\mathbb{B}_{d}^{r}$. The group $\mathbb{B}_{r}$ acts as follows:

$$
\left(\tau_{1}, \ldots, \tau_{r}\right)^{\sigma_{i}}:=\left(\tau_{1}, \ldots, \tau_{i-1}, \tau_{i+1}, \tau_{i+1} \tau_{i} \tau_{i+1}^{-1}, \tau_{i+2}, \ldots, \tau_{r}\right)
$$

where $\sigma_{i} \in \mathbb{B}_{r}, i=1, \ldots, r-1$, is a canonical generator and $\left(\tau_{1}, \ldots, \tau_{r}\right) \in \mathbb{B}_{d}^{r}$. This action of a braid in $\mathbb{B}_{r}$ is classically called a Hurwitz move. The second action is given by $\mathbb{B}_{n}$ and is defined by simultaneous conjugation:

$$
\left(\tau_{1}, \ldots, \tau_{r}\right)^{\beta}:=\left(\tau_{1}^{\beta}, \ldots, \tau_{r}^{\beta}\right),
$$

where $\beta \in \mathbb{B}_{d}$ and $\left(\tau_{1}, \ldots, \tau_{r}\right) \in \mathbb{B}_{d}^{r}$. These actions commute with each other and hence they define a new action of $\mathbb{B}_{r} \times \mathbb{B}_{d}$.

A different choice of geometric basis of $\pi_{1}(\mathbb{C} \backslash \mathscr{D} ; \star)$ modifies $\left(\tau_{1}, \ldots, \tau_{r}\right)$ as a Hurwitz move. A different choice of $\beta, \star$ or $\Delta$ modifies $\left(\tau_{1}, \ldots, \tau_{r}\right)$ by simultaneous conjugation. Moreover, any element in the orbit of $\left(\tau_{1}, \ldots, \tau_{r}\right)$ by the action of $\mathbb{B}_{r} \times \mathbb{B}_{d}$ can be obtained by suitable choices of $\Delta, \star, \beta$ and a geometric basis of $\pi_{1}(\mathbb{C} \backslash \mathscr{D} ; \star)$.

Definition 2.4. The braid monodromy of the triple $(\mathcal{C}, L, P)$ is the orbit of $\left(\tau_{1}, \ldots, \tau_{r}\right)$ by the action of $\mathbb{B}_{r} \times \mathbb{B}_{d}$.

Remark 2.5. As in [3], if we replace a geometric basis of $\pi_{1}(\mathbb{C} \backslash \mathscr{D} ; \star)$ by a pseudogeometric basis (see Remark [1.6), we obtain also an element of $\mathbb{B}_{d}^{r}$ in the braid monodromy of the triple.

We are going to define these concepts in a more general way. Let $G$ be a group and let $r$ be a positive integer. Let us consider the sets $G^{r}$ and $G^{r} / G$, the latter being the quotient of $G^{r}$ by the diagonal action of $G$ by conjugation. The braid group $\mathbb{B}_{r}$ acts on $G^{r}$ by the so-called Hurwitz action:

$$
\left(g_{1}, \ldots, g_{r}\right)^{\sigma_{j}}:=\left(g_{1}, \ldots, g_{j-1}, g_{j+1}, g_{j+1} g_{j} g_{j+1}^{-1}, g_{j+2}, \ldots, g_{r}\right), 1 \leq j<r,
$$

where $g_{1}, \ldots, g_{r} \in G$. Since Hurwitz and conjugation actions commute, $\mathbb{B}_{r}$ also acts on $G^{r} / G$.

Let us denote by $\mathscr{N}_{r}(G)$ (resp. $\left.\mathscr{M}_{r}(G)\right)$ the quotient of $G^{r}$ (resp. $G^{r} / G$ ) by the Hurwitz action. The index $r$ will be omitted if no ambiguity seems likely to arise. 
Definition 2.6. The elements of $\mathscr{M}_{r}(G)$ after the above discussion will be called $G$-monodromies (of order $r$ ).

Note that the braid monodromy of a horizontal triple $(\mathcal{C}, L, P)$ of degree $d$ possessing $r$ non-transversal vertical lines is an element in $\mathscr{M}_{r}\left(\mathbb{B}_{d}\right)$.

Remark 2.7. This point of view is inspired by the work of E. Brieskorn on automorphic sets [6]. We restrict our attention to automorphic sets defined by conjugation on groups. We have also modified the conjugation action defined in 6 .

We now state the main result of $[3$ which will be used later.

Theorem 2.8. Let $\left(\mathcal{C}_{1}, L, P\right)$ and $\left(\mathcal{C}_{2}, L, P\right)$ be two horizontal triples. Let $F: \mathbb{P}^{2} \rightarrow$ $\mathbb{P}^{2}$ be an orientation-preserving homeomorphism such that:

(i) $F([0: 0: 1])=[0: 0: 1]$, i.e., $F$ respects the base point of the fibration.

(ii) $F(L)=L$ preserving orientations.

(iii) $F\left(\mathcal{C}_{1}^{\varphi}\right)=\mathcal{C}_{2}^{\varphi}$ preserving orientations.

Then $\left(\mathcal{C}_{1}, L, P\right)$ and $\left(\mathcal{C}_{2}, L, P\right)$ have the same braid monodromy.

This theorem will be used to compare curves in $\mathbf{M}_{1}$ and $\mathbf{M}_{2}$. We must compute their braid monodromies and find suitable invariants in order to be able to compare them. We now define such invariants.

Definition 2.9. Let $\mathfrak{g}:=\left(g_{1}, \ldots, g_{r}\right) \in G^{r}$. The pseudo-Coxeter element associated with $\mathfrak{g}$ is defined as $c(\mathfrak{g}):=g_{r} \cdot \ldots \cdot g_{1}$.

Note that a pseudo-Coxeter element is also well defined in $\mathscr{N}(G)$ and its conjugation class is well defined in $\mathscr{M}(G)$.

Let $\Psi: G_{1} \rightarrow G_{2}$ be a group homomorphism. It induces mappings $\Psi_{\mathscr{N}}$ : $\mathscr{N}\left(G_{1}\right) \rightarrow \mathscr{N}\left(G_{2}\right)$ and $\Psi_{\mathscr{M}}: \mathscr{M}\left(G_{1}\right) \rightarrow \mathscr{M}\left(G_{2}\right)$ in a functorial way.

Definition 2.10. Let $(\mathcal{C}, L, P)$ be a horizontal triple of degree $d$ possessing $r$ nontransversal lines and let $\Psi: \mathbb{B}_{d} \rightarrow G$ be a representation of $\mathbb{B}_{d}$ onto a group $G$. The $(\Psi, G)$-monodromy of $(\mathcal{C}, L, P)$ is defined as the image of the braid monodromy of $(\mathcal{C}, L, P)$ in $\mathscr{M}_{r}(G)$ by $\Psi_{\mathscr{M}}$.

Proposition 2.11. Let $\left(\mathcal{C}_{1}, L, P\right)$ and $\left(\mathcal{C}_{2}, L, P\right)$ be horizontal triples of degree $d$ having $r$ non-transversal vertical lines. Let $\Psi: \mathbb{B}_{d} \rightarrow G$ be a representation. If the $(\Psi, G)$-monodromies of $\left(\mathcal{C}_{1}, L, P\right)$ and $\left(\mathcal{C}_{2}, L, P\right)$ are not equal, then there is no orientation-preserving homeomorphism satisfying the properties of Theorem 2.8 .

Proof. This is a straightforward consequence of Theorem 2.8

If $G$ is a finite group, then $\mathscr{M}(G)$ is a finite set and hence, up to computational capacity, knowing the braid monodromies of two given curves would allow us to compare their $(\Psi, G)$-monodromies.

Algorithm 2.12. We have implemented an algorithm on GAP4 [10]. Its input consists of braid monodromies of two curves and a finite representation of the braid group. Its output confirms whether or not the $(\Psi, G)$-monodromies are equal. We sketch the general lines of the algorithm:

(i) Compute representatives of the braid monodromies of the triples $\left(\mathcal{C}_{1}, L, P\right)$ and $\left(\mathcal{C}_{2}, L, P\right)$. We can order the representatives such that correlative elements are conjugate in $\mathbb{B}_{d}$; this is possible if the curves have the same 
combinatorics. We denote $\tilde{\mathbb{B}}_{r}$ the subgroup of Hurwitz moves which preserve this order. Then we compute representatives $n_{G}\left(\mathcal{C}_{i}\right), i=1,2$, of the $(\Psi, G)$-monodromies.

(ii) Compute their pseudo-Coxeter elements $c_{G}\left(\mathcal{C}_{1}\right), c_{G}\left(\mathcal{C}_{2}\right) \in G$. If they are not conjugate, braid monodromies are not equal and hence we are done. If they are conjugate to each other, choose an element $g \in G$ such that $c_{G}\left(\mathcal{C}_{2}\right)^{g}=c_{G}\left(\mathcal{C}_{1}\right)=: h$. We replace $n_{G}\left(\mathcal{C}_{2}\right)$ by $n_{G}\left(\mathcal{C}_{2}\right)^{g}$. Let $H$ be the centralizer of $h$ in $G$. Let $m_{G}\left(\mathcal{C}_{i}\right)$ be the orbit of $n_{G}\left(\mathcal{C}_{i}\right)$ by the action of $H$, $i=1,2$.

(iii) Instead of computing the orbit of $n_{G}\left(\mathcal{C}_{1}\right)$ by the action of $\mathbb{B}_{r} \times G$, we compute the orbit of $m_{G}\left(\mathcal{C}_{1}\right)$ by the action of $\tilde{\mathbb{B}}_{r} \times H$. In fact, we check at each point that the new elements of this orbit are equal to $m_{G}\left(\mathcal{C}_{2}\right)$.

(iv) If $m_{G}\left(\mathcal{C}_{2}\right)$ is not in this orbit, then the two $(\Psi, G)$-monodromies are not equal.

Strategy 2.13. In order to distinguish braid monodromies for two horizontal triples $\left(\mathcal{C}_{1}, L, P\right)$ and $\left(\mathcal{C}_{2}, L, P\right)$ of the same degree, we propose the following procedure:

(a) Verify whether or not both the affine and projective curves have the same combinatorics. If not, we are done.

(b) Otherwise, compute the fundamental group of $X_{\mathcal{C}_{i}}$ and verify whether or not they are isomorphic. In the second case, we are done.

(c) If either the groups are isomorphic or this cannot be determined, then compute the braid monodromy groups which is the conjugacy class of the group generated by the elements in a representative of a braid monodromy. If conjugacy classes do not coincide, we are done.

(d) If they do, then one can still verify whether or not any of the elements that transforms one representative group into the other by conjugation also transforms one pseudo-Coxeter element into the other by conjugation. If they do not, we are done.

(e) If one cannot determine whether or not the monodromy groups are conjugate, then Libgober invariants (which provide easy-to-compare polynomials) or the sequence of characteristic varieties might help to distinguish the fundamental groups.

(f) If either the previous steps do not work or they are hard to check, one can try the methods described in this section.

\section{Construction of Curves in M}

Curves in $\mathbf{M}:=\mathbf{M}\left(\mathbb{E}_{6}, \mathbb{A}_{7}, \mathbb{A}_{3}, \mathbb{A}_{2}, \mathbb{A}_{1} ; 6\right)$ will be constructed following the ideas in [4] (see Theorem 1 in the Introduction). Next, we provide an outline of the method used:

- Start assuming $\mathcal{C}$ is a curve in $\mathbf{M}$. We will refer to the singular points of $\mathcal{C}$ by their singular type.

- Perform a standard Cremona transformation with base points $\mathbb{E}_{6}, \mathbb{A}_{3}$ and $\mathbb{A}_{2}$ (note that they are not aligned).

- The strict transform of the quintic (resp. the line) in $\mathcal{C}$ is a nodal cubic $\mathcal{N}$ (resp. a conic $\mathcal{Q}$ ). The strict transform of $\mathbb{E}_{6}$ is an inflection point and the strict transform of $\mathbb{A}_{3}$ is the double point of $\mathcal{N}$. There are three points in $\mathcal{Q} \cap \mathcal{N}$. Around two of them (the images of $\mathbb{A}_{7}$ and $\mathbb{A}_{1}$ ) the Cremona 
transformation is a local isomorphism. The remaining is a node and lies on the tangent line to $\mathcal{N}$ at the strict transform of $\mathbb{A}_{2}$.

- Using the group structure of the smooth part of $\mathcal{N}$ (and Maple7) it is not difficult to prove that, up to projective transformation, only two cases are possible.

- Considering the inverse of the Cremona transformation it turns out that any curve in $\mathbf{M}$ is projectively equivalent to exactly one of the following two projective curves $\mathcal{C}_{\beta}:=\left\{f_{\beta} g_{\beta}=0\right\}, \beta^{2}=2$, where:

$$
\begin{aligned}
f_{\beta}(x, y, z):= & y^{2} z^{3}+(303-216 \beta) y z^{2} x^{2}+(-636+450 \beta) y z x^{3} \\
& +(-234 \beta+331) y x^{4}+(-18 \beta+27) z x^{4}+(18 \beta-26) x^{5},
\end{aligned}
$$

and

$$
g_{\beta}(x, y, z):=y+\left(\frac{10449}{196}-\frac{3645}{98} \beta\right) z+\left(-\frac{432}{7}+\frac{297}{7} \beta\right) x .
$$

The line $L:=\{z=0\}$ is the tangent line to both curves $\mathcal{C}_{\beta}$ at the $\mathbb{E}_{6}$-point, which is $P:=[0: 1: 0]$. We take the affine plane of coordinates $(x, y)$ and consider the projection $\pi(x, y)=x$. Since mult $_{\mathbb{E}_{6}}\left(\mathcal{C}_{\beta}\right)=3$, one has $\operatorname{deg}_{\pi}\left(\mathcal{C}_{\beta}\right)=3$. There are 4 non-transversal vertical lines corresponding to the singular points of types $\mathbb{A}_{7}$, $\mathbb{A}_{3}, \mathbb{A}_{2}$, and $\mathbb{A}_{1}$. There is also an ordinary tangent vertical line intersecting $\mathcal{C}_{\beta}$ at a point of tangency denoted by $\mathbb{A}_{0}$. The values for $x$ at these lines are shown in Table 1.

\section{TABle 1.}

\begin{tabular}{|c|c|c|c|c|}
\hline $\mathbb{A}_{7}$ & $\mathbb{A}_{3}$ & $\mathbb{A}_{2}$ & $\mathbb{A}_{1}$ & $\mathbb{A}_{0}$ \\
\hline$\frac{90+9 \beta}{98}$ & 0 & 1 & $\frac{18+27 \beta}{56}$ & $-\frac{45+36 \beta}{7}$ \\
\hline
\end{tabular}

Let us denote by $\mathcal{C}_{\beta}^{\text {aff }}$ the affine curve $f_{\beta}(x, y, 1) g_{\beta}(x, y, 1)=0$. In both cases $\mathscr{D}_{\beta} \subset \mathbb{R}$ and has cardinality five. Let us choose $\star \in \mathbb{R}, \star \gg 0$ and consider $\pi_{1}\left(\mathbb{C} \backslash \mathscr{D}_{\beta} ; \star\right)$. We order $\mathscr{D}_{\beta}:=\left\{x_{1}^{\beta}, \ldots, x_{5}^{\beta}\right\}$, such that $x_{1}^{\beta}>\cdots>x_{5}^{\beta}$.

Figures 2 and 3 show the real parts of $\mathcal{C}_{\sqrt{2}}^{\text {aff }}$ and $\mathcal{C}_{-\sqrt{2}}^{\text {aff }}$. The dotted paths represent the real parts of the imaginary solutions and the thick point is the tacnode. The branch at infinity corresponding to the $\mathbb{E}_{6}$-point is represented in both cases by the branch marked with an arrow.

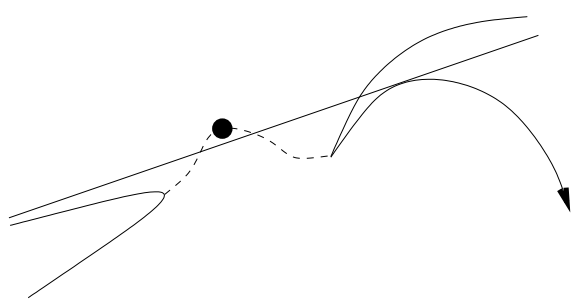

FiguRE 2. Real part of $\mathcal{C}_{\sqrt{2}}^{\text {aff }}$ 


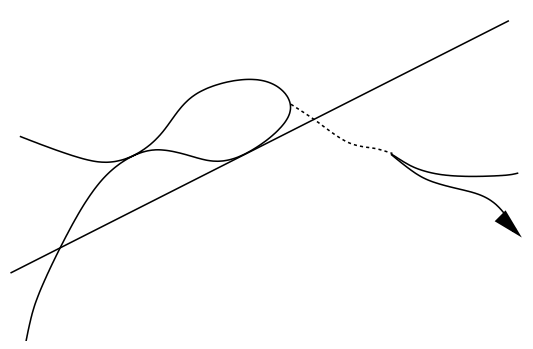

FIgURE 3. Real part of $\mathcal{C}_{-\sqrt{2}}^{\text {aff }}$

Remark 3.1. In order to obtain Figures 2 and 3, one must first identify the singular points of the projection. Next one must compute the intersection of the curve with both the fixed generic line and the non-transversal lines. Once this is done, the task becomes straightforward.

The computation of the braid monodromy is performed as in 44. We recall the main ideas. For $j=1, \ldots, 5, \beta= \pm \sqrt{2}$ we denote:

- by $\nu_{j}^{\beta}$ the paths in the real axis starting at $x_{j-1}^{\beta}-\varepsilon$ and ending at $x_{j}^{\beta}+\varepsilon$ as shown in Figure 4, where $\varepsilon>0$ is small enough and $x_{0}^{\beta}:=\star$. We associate braids with different ends to these paths which can be identified to elements in $\mathbb{B}_{3}$ using the lexicographic bijections of Remark 1.8. We also follow the conventions explained in that remark to determine the braids when couples of complex conjugate solutions exist. In our case, since only one such couple may exist we only need to know their common real parts.

- by $\delta_{j}^{\beta}$ the loops based at $x_{j}^{\beta}+\varepsilon$ which run counterclockwise to the circle of center $x_{j}^{\beta}$ and radius $\varepsilon$ as shown in Figure 4. The closed braids associated with these loops are also identified by means of the lexicographic isomorphism with braids in $\mathbb{B}_{3}$. The result depends on the ordering of the points and on the local Puiseux expansions of the singular points of the projections. For example, when considering the braid associated with a point of type $\mathbb{A}_{2}$ with Puiseux expansion $y=x^{\frac{3}{2}}+\ldots$, which has the biggest real part in the singular fiber, the associated braid is $\sigma_{1}^{3}$.

- by $\left(\delta_{j}^{\beta}\right)^{+}$the the first half part of $\delta_{j}^{\beta}$ as shown in Figure 4. In order to obtain the braids associated with these paths one must consider the braids associated with $\delta_{j}^{\beta}$ and the conventions of Remark 1.8. In the example above, when the path arrives at $x_{j}^{\beta}-\varepsilon$, the roots are complex conjugate. Applying the conventions of Remark 1.8, we obtain the braid $\sigma_{1}$. If the Puiseux expansion is $y=i x^{\frac{3}{2}}+\ldots$, we obtain $\sigma_{1}^{2}$.

Note that

$$
\alpha_{k}^{\beta}:=\left(\prod_{j=1}^{k-1}\left(\nu_{j}^{\beta}\left(\delta_{j}^{\beta}\right)^{+}\right) \nu_{k}^{\beta}\right) * \delta_{k}^{\beta}
$$

is a geometric basis of $\pi_{1}\left(\mathbb{C} \backslash \mathscr{D}_{\beta} ; \star\right)$ (the operation $*$ was defined in $\left.\$ \mathbb{1}\right)$. If we denote by $\tilde{\alpha}_{j}^{\beta}$ the meridian about the non-generic line passing through the point 


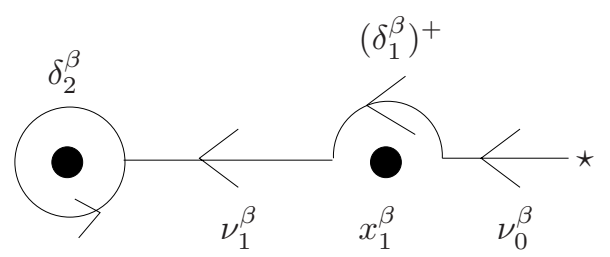

Figure 4. Paths on $\mathbb{C} \backslash \mathscr{D}_{\beta}$

$\mathbb{A}_{j}$, we obtain

$$
\alpha_{1}^{\sqrt{2}}=\tilde{\alpha}_{7}^{\sqrt{2}}, \quad \alpha_{2}^{\sqrt{2}}=\tilde{\alpha}_{1}^{\sqrt{2}}, \quad \alpha_{3}^{\sqrt{2}}=\tilde{\alpha}_{2}^{\sqrt{2}}, \quad \alpha_{4}^{\sqrt{2}}=\tilde{\alpha}_{3}^{\sqrt{2}}, \quad \alpha_{5}^{\sqrt{2}}=\tilde{\alpha}_{0}^{\sqrt{2}}
$$

and

$$
\alpha_{1}^{-\sqrt{2}}=\tilde{\alpha}_{2}^{-\sqrt{2}}, \quad \alpha_{2}^{-\sqrt{2}}=\tilde{\alpha}_{0}^{-\sqrt{2}}, \quad \alpha_{3}^{-\sqrt{2}}=\tilde{\alpha}_{7}^{-\sqrt{2}}, \quad \alpha_{4}^{-\sqrt{2}}=\tilde{\alpha}_{3}^{-\sqrt{2}}, \quad \alpha_{5}^{-\sqrt{2}}=\tilde{\alpha}_{1}^{-\sqrt{2}} .
$$

Note that the subindex in $\tilde{\alpha}_{j}^{\beta}$ refers to the topological type of the singular point of the projection.

The results for $\mathcal{C}_{\sqrt{2}}^{\text {aff }}$ and $\mathcal{C}_{-\sqrt{2}}^{\text {aff }}$ are shown in Table 2,

TABLe 2 .

\begin{tabular}{|c|c||c|c|}
\hline$\nu_{1}^{\sqrt{2}}$ & 1 & $\nu_{1}^{-\sqrt{2}}$ & 1 \\
\hline$\delta_{1}^{\sqrt{2}}$ & $\sigma_{2}^{8}$ & $\delta_{1}^{-\sqrt{2}}$ & $\sigma_{2}^{3}$ \\
\hline$\left(\delta_{1}^{\sqrt{2}}\right)^{+}$ & $\sigma_{2}^{4}$ & $\left(\delta_{1}^{-\sqrt{2}}\right)^{+}$ & $\sigma_{2}^{3}$ \\
\hline$\nu_{2}^{\sqrt{2}}$ & 1 & $\nu_{2}^{-\sqrt{2}}$ & $\sigma_{1}^{-1} \sigma_{2}$ \\
\hline$\delta_{2}^{\sqrt{2}}$ & $\sigma_{1}^{2}$ & $\delta_{2}^{-\sqrt{2}}$ & $\sigma_{1}$ \\
\hline$\left(\delta_{2}^{\sqrt{2}}\right)^{+}$ & $\sigma_{1}$ & $\left(\delta_{2}^{-\sqrt{2}}\right)^{+}$ & $\sigma_{1}$ \\
\hline$\nu_{3}^{\sqrt{2}}$ & 1 & $\nu_{3}^{-\sqrt{2}}$ & 1 \\
\hline$\delta_{3}^{\sqrt{2}}$ & $\sigma_{2}^{3}$ & $\delta_{3}^{-\sqrt{2}}$ & $\sigma_{2}^{8}$ \\
\hline$\left(\delta_{3}^{\sqrt{2}}\right)^{+}$ & $\sigma_{2}$ & $\left(\delta_{3}^{-\sqrt{2}}\right)^{+}$ & $\sigma_{2}^{4}$ \\
\hline$\nu_{4}^{\sqrt{2}}$ & $\sigma_{1}^{-1} \sigma_{2}$ & $\nu_{4}^{-\sqrt{2}}$ & 1 \\
\hline$\delta_{4}^{\sqrt{2}}$ & $\sigma_{1}^{4}$ & $\delta_{4}^{-\sqrt{2}}$ & $\sigma_{1}^{4}$ \\
\hline$\left(\delta_{4}^{\sqrt{2}}\right)^{+}$ & $\sigma_{1}^{2}$ & $\left(\delta_{4}^{-\sqrt{2}}\right)^{+}$ & $\sigma_{1}^{2}$ \\
\hline$\nu_{5}^{\sqrt{2}}$ & $\sigma_{2}^{-1} \sigma_{1}$ & $\nu_{5}^{-\sqrt{2}}$ & 1 \\
\hline$\delta_{5}^{\sqrt{2}}$ & $\sigma_{2}$ & $\delta_{5}^{-\sqrt{2}}$ & $\sigma_{2}^{2}$ \\
\hline
\end{tabular}


Applying standard simplifications of braids, the following braid monodromies for $\left(\mathcal{C}_{\sqrt{2}}, L, P\right)$ and $\left(\mathcal{C}_{-\sqrt{2}}, L, P\right)$ are obtained:

$$
\begin{array}{ll}
\tilde{\alpha}_{7}^{\sqrt{2}} \mapsto \sigma_{2}^{8} & \tilde{\alpha}_{2}^{-\sqrt{2}} \mapsto \sigma_{2}^{3} \\
\tilde{\alpha}_{1}^{\sqrt{2}} \mapsto \sigma_{2}^{4} * \sigma_{1}^{2} & \tilde{\alpha}_{0}^{-\sqrt{2}} \mapsto \sigma_{2} \sigma_{1}^{-1} \sigma_{2} * \sigma_{1} \\
\tilde{\alpha}_{2}^{\sqrt{2}} \mapsto \sigma_{2}^{3} * \sigma_{1}^{3} & \tilde{\alpha}_{7}^{-\sqrt{2}} \mapsto \sigma_{2} * \sigma_{1}^{8} \\
\tilde{\alpha}_{3}^{\sqrt{2}} \mapsto \sigma_{2} * \sigma_{1}^{4} & \tilde{\alpha}_{3}^{-\sqrt{2}} \mapsto \sigma_{1}^{-2} * \sigma_{2}^{4} \\
\tilde{\alpha}_{0}^{\sqrt{2}} \mapsto \sigma_{1}^{-3} * \sigma_{2} & \tilde{\alpha}_{1}^{-\sqrt{2}} \mapsto \sigma_{1}^{-3} * \sigma_{2}^{2} .
\end{array}
$$

Let us consider $n_{\sqrt{2}}:=\left[\sigma_{2}^{8}, \sigma_{2}^{4} * \sigma_{1}^{2}, \sigma_{2}^{3} * \sigma_{1}^{3}, \sigma_{2} * \sigma_{1}^{4}, \sigma_{1}^{-3} * \sigma_{2}\right] \in \mathbb{B}_{3}^{5}$. Its image $m_{\sqrt{2}}$ in $\mathscr{M}\left(\mathbb{B}_{3}\right)$ is the braid monodromy of $\left(\mathcal{C}_{\sqrt{2}}, L, P\right)$. Analogously, consider $n_{-\sqrt{2}}:=$ $\left[\sigma_{2}^{3},\left(\sigma_{2} \sigma_{1}^{-1} \sigma_{2}\right) * \sigma_{1}, \sigma_{2} * \sigma_{1}^{8}, \sigma_{1}^{-2} * \sigma_{2}^{4}, \sigma_{1}^{-3} * \sigma_{2}^{2}\right]$. Its image $m_{-\sqrt{2}}$ in $\mathscr{M}\left(\mathbb{B}_{3}\right)$ is the braid monodromy of $\left(\mathcal{C}_{-\sqrt{2}}, L, P\right)$.

Applying the Zariski-Van Kampen Theorem, the fundamental group $\pi_{1}\left(X_{\mathcal{C}_{\beta}}\right)$ is obtained. In order to obtain $\pi_{1}\left(\mathbb{P}^{2} \backslash \mathcal{C}_{\beta}\right)$ one must kill a meridian about the line $L$, which can be done by taking into account the behavior of the branches at infinity. Using GAP4 [10, we obtain that both fundamental groups are isomorphic to $\mathbb{Z} \times S L(2 ; \mathbb{Z} / 7 \mathbb{Z})$. Moreover, there is an isomorphism preserving meridians, i.e., the image of a meridian in $\mathcal{C}_{\sqrt{2}}$ is a meridian in $\mathcal{C}_{-\sqrt{2}}$.

Theorem 3.2. The braid monodromies of $\left(\mathcal{C}_{\sqrt{2}}, L, P\right)$ and $\left(\mathcal{C}_{-\sqrt{2}}, L, P\right)$ are not equal.

Proof. The methods (国), (b) and (ㄷ) in Strategy 2.13 do not distinguish the braid

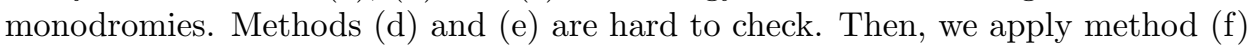
using the representation

$$
\mathbb{B}_{3} \stackrel{\psi}{\rightarrow} S L(2 ; \mathbb{Z}) \stackrel{j}{\rightarrow} S L(2 ; \mathbb{Z} / 32 \mathbb{Z})=: G,
$$

where $\psi$ is defined by

$$
\psi\left(\sigma_{1}\right):=\left(\begin{array}{rr}
1 & 0 \\
-1 & 1
\end{array}\right), \quad \psi\left(\sigma_{2}\right):=\left(\begin{array}{ll}
1 & 1 \\
0 & 1
\end{array}\right)
$$

and $j$ is given by projecting the coefficients. Let $\Psi:=\psi \circ j: \mathbb{B}_{3} \rightarrow G$ be the composition of these maps. The image of $n_{\sqrt{2}}$ by $\Psi$ is a list

$$
n_{\sqrt{2}}(G):=\left[\left(\begin{array}{ll}
1 & 8 \\
0 & 1
\end{array}\right),\left(\begin{array}{ll}
25 & 0 \\
30 & 9
\end{array}\right),\left(\begin{array}{ll}
24 & 27 \\
29 & 10
\end{array}\right),\left(\begin{array}{ll}
30 & 1 \\
23 & 4
\end{array}\right),\left(\begin{array}{ll}
13 & 4 \\
28 & 21
\end{array}\right)\right],
$$

and its image $m_{\sqrt{2}}(G)$ in $G^{5} / G$ is a representative of $\mathscr{M}_{\sqrt{2}}(G)$, the $(\Psi, G)$-monodromy of $\mathcal{C}_{\sqrt{2}}$. The orbit $\mathscr{M}_{\sqrt{2}}(G)$ contains exactly 15360 elements in $G^{5} / G$. All of this can also be done for $\beta=-\sqrt{2}$. Let us explain how the output of the GAP4-format progran 11 can be used to prove that $\mathscr{M}_{\sqrt{2}}(G) \cap \mathscr{M}_{-\sqrt{2}}(G)=\emptyset$. The execution of the program took a couple of minutes.

Since all the non-transversal vertical lines have different topological behavior in this example, the group $\tilde{\mathbb{B}}_{5}$ of Algorithm 2.12(i) is the pure braid group $\mathbb{P}_{5} \subset \mathbb{B}_{5}$. We compute the orbit $\mathbb{P}_{\sqrt{2}}(G)$ of $m_{\sqrt{2}}(G)$ by the action of $\mathbb{P}_{5}$. Each element of $\mathbb{P}_{\sqrt{2}}(G) \subset G^{5} / G$ is described by a random representative in $G^{5}$.

Since $\mathbb{B}_{5}$ acts transitively on the set of geometric bases, we choose a braid $\tau \in \mathbb{B}_{5}$ such that the elements of the geometric basis obtained from $\tilde{\alpha}_{k}^{-\sqrt{2}}, k=2,0,7,3,1$,

\footnotetext{
${ }^{1}$ Available at http://riemann.unizar.es/geotop/pub/gap-programs .
} 
by the action of $\tau$, are ordered in the same manner as those of the given geometric basis for $\mathcal{C}_{\sqrt{2}}$, i.e.,

- the first one is a meridian of the line passing through $\mathbb{A}_{7}$;

- the second one is a meridian of the line passing through $\mathbb{A}_{1}$;

- and so on ...

Let $\tilde{n}_{-\sqrt{2}}(G):=n_{-\sqrt{2}}(G)^{\tau}$ and let us denote by $c_{\sqrt{2}}(G)$ and $c_{-\sqrt{2}}(G)$ the pseudoCoxeter elements of $n_{\sqrt{2}}(G)$ and $\tilde{n}_{-\sqrt{2}}(G)$, respectively. They are conjugated in $G$. In fact, they come from pseudo-Coxeter elements which are conjugated in $\mathbb{B}_{3}$. Let $h \in G$ be such that

$$
c_{-\sqrt{2}}(G)^{h}=c_{\sqrt{2}}(G):=g .
$$

Let $\hat{n}_{-\sqrt{2}}(G) \in G^{5}$ be the element obtained from $\tilde{n}_{-\sqrt{2}}(G)$ after simultaneous conjugation by $h$. Note that the pseudo-Coxeter element of $\hat{n}_{-\sqrt{2}}(G)$ is $g$. Let $C_{g}$ be the centralizer of $g$ in $G$ and let us denote by $\Omega$ the orbit of $\hat{n}_{-\sqrt{2}}(G)$ by the conjugation action of $C_{g}$. In order to prove that $\mathscr{M}_{\sqrt{2}}(G) \cap \mathscr{M}_{-\sqrt{2}}(G)=\emptyset$ it is enough to see that no element of $\Omega$ is in the orbit.

Finally, we are in the position to prove Theorem 2

Proof of Theorem 2, Let $\mathcal{C}_{1}$ and $\mathcal{C}_{2}$ denote $\mathcal{C}_{\sqrt{2}}$ and $\mathcal{C}_{-\sqrt{2}}$, respectively. By Theorems 3.2 and 2.8 there is no homeomorphism $\left(\mathbb{P}^{2}, \mathcal{C}_{1}^{\varphi}\right) \rightarrow\left(\mathbb{P}^{2}, \mathcal{C}_{2}^{\varphi}\right)$ preserving orientations on $\mathbb{P}^{2}, \mathcal{C}_{1}$ and $\mathcal{C}_{2}$. Note also that any homeomorphism should respect the projection point and the line at infinity by local considerations.

Since both curves have real equations, they are invariant by complex conjugation which preserves orientations on $\mathbb{P}^{2}$ but exchanges orientations on the curves. Hence, there is no homeomorphism $\left(\mathbb{P}^{2}, \mathcal{C}_{1}^{\varphi}\right) \rightarrow\left(\mathbb{P}^{2}, \mathcal{C}_{2}^{\varphi}\right)$ preserving the orientation on $\mathbb{P}^{2}$ and reversing it on $\mathcal{C}_{1}$ and $\mathcal{C}_{2}$.

Note that the local braids associated with singular germs of curves always turn in the same direction. This means that any such braid can be written as a word in standard generators whose exponents are necessarily positive (such braids are commonly called positive). Therefore, it is not possible to have a homeomorphism $\left(\mathbb{P}^{2}, \mathcal{C}_{1}^{\varphi}\right) \rightarrow\left(\mathbb{P}^{2}, \mathcal{C}_{2}^{\varphi}\right)$ preserving the orientation on $\mathbb{P}^{2}$ and only on some, but not all, of the components of $\mathcal{C}_{i}$.

\section{Other EXAmples}

Remark 4.1. All these examples appear in Yang's list of sextic curves with simple singularities (see [18) and they all have maximal Milnor number 19.

As a general overview of the techniques that can be applied using braid monodromy of triples and a refinement of Theorem 2.8. we present only some of the most characteristic examples that have been found so far. We follow the steps in Strategy 2.13. step (国) is trivially verified and step (b) gives no information, since all the involved fundamental groups are Abelian (the latter can be obtained after calculating braid monodromy representatives).

Example 4.2. The space $\mathbf{M}\left(\mathbb{E}_{8}, \mathbb{E}_{7}, \mathbb{A}_{4} ; 6\right)$ has exactly two points and we can choose representatives $\mathcal{C}_{i}$ and $\mathcal{C}_{-i}$ with equations in $\mathbb{Q}(i)[x, y, z]$ :

$$
\begin{aligned}
f_{s}(x, y, z):= & (5+s) x^{6}+(-11-2 s) y x^{5}+z(-12+28 s) x^{5}+(16-52 s) x^{4} y z \\
& +(-52-36 s) x^{4} z^{2}+(108+24 s) x^{3} y z^{2}+(32-32 s) x^{3} z^{3} \\
& +(-48+16 s) y^{2} x^{2} z^{2}+96 s y x^{2} z^{3}+(-48-48 s) y^{2} x z^{3}+16 z^{3} y^{3}
\end{aligned}
$$


where $s^{2}+1=0$. These curves are obviously homeomorphic by complex conjugation. Let us consider the triples associated with the point $P=[0: 1: 0]$ of type $\mathbb{E}_{8}$ and the tangent line $L=\{z=0\}$ at $P$. Using the Maple7-format program developed by the third author in 7, representatives of these braid monodromies are found (see Table 3). Due to complex conjugation, one representative determines the other as explained below.

TABLE 3.

\begin{tabular}{|c|c|c|c|}
\hline$s=i$ & $\sigma_{2} \sigma_{1} \sigma_{2} \sigma_{1}^{2} \sigma_{2}^{2} \sigma_{1} \sigma_{2}$ & $\sigma_{2} * \sigma_{1}^{5}$ & $\left(\sigma_{1}^{-1} \sigma_{2}\right) * \sigma_{1}$ \\
\hline$s=-i$ & $\sigma_{2} \sigma_{1} \sigma_{2}^{2} \sigma_{1}^{2} \sigma_{2} \sigma_{1} \sigma_{2}$ & $\sigma_{2}^{5}$ & $\sigma_{2}^{2} * \sigma_{1}$ \\
\hline
\end{tabular}

Lemma 4.3. Let us suppose that $\left(\tau_{1}, \ldots, \tau_{r}\right) \in \mathbb{B}_{d}^{r}$ represents the braid monodromy of a horizontal triple $(\mathcal{C}, L, P)$, where $P=[0: 1: 0]$ and $L=\{z=0\}$. Let $(\overline{\mathcal{C}}, L, P)$ be the horizontal triple obtained by complex conjugation.

Then $\left(\bar{\tau}_{r}^{-1}, \ldots, \bar{\tau}_{1}^{-1}\right) \in \mathbb{B}_{d}^{r}$ represents the braid monodromy of $(\overline{\mathcal{C}}, L, P)$, where, given $\tau \in \mathbb{B}_{d}$ as a word $w\left(\sigma_{1}, \ldots, \sigma_{d-1}\right)$, the braid $\bar{\tau}$ is the word $w\left(\sigma_{1}^{-1}, \ldots, \sigma_{d-1}^{-1}\right)$.

Table 3 is obtained by combining this lemma with a series of Hurwitz moves. Using a GAP4-format program we find that the $(\Psi, S L(2 ; \mathbb{Z} / 5 \mathbb{Z}))$-monodromies of these triples are not equal. The restricted orbits to the pure braid group (the second strategy followed in the proof of Theorem 3.2) have only one element. This implies that no oriented homeomorphism exists between $\left(\mathbb{P}^{2}, \mathcal{C}_{i}^{\varphi} \cup L\right)$ and $\left(\mathbb{P}^{2}, \mathcal{C}_{-i}^{\varphi} \cup L\right)$.

Example 4.4. The space $\mathbf{M}\left(\mathbb{E}_{8}, \mathbb{A}_{6}, \mathbb{A}_{4}, \mathbb{A}_{1} ; 6\right)$ consists of three points and we can choose representatives with equations depending on $s$ where $28 s^{3}+72 s^{2}+42 s-7=0$. Equations are rather long and can be made available upon request. This equation has one real solution $s_{1}$ and two complex conjugate solutions $s_{2}$ and $\bar{s}_{2}$. We consider $P$ and $L$ as in Example 4.2. Using the program provided in [7] we compute the braid monodromy representatives for $s=s_{1}, s_{2}$ (see Table 4). For $s=\bar{s}_{2}$ we apply Lemma 4.3

TABLE 4.

\begin{tabular}{|c|c|c|c|c|}
\hline$s=s_{1}$ & $\sigma_{1}^{2}$ & $\sigma_{2}^{7}$ & $\left(\sigma_{2}^{2} \sigma_{1}^{-2}\right) * \sigma_{2}$ & $\sigma_{2}^{2} * \sigma_{1}^{5}$ \\
\hline$s=s_{2}$ & $\sigma_{2} * \sigma_{1}^{2}$ & $\sigma_{1}^{7}$ & $\sigma_{1}^{3} * \sigma_{2}$ & $\sigma_{1}^{5}$ \\
\hline
\end{tabular}

Following (ㄷ) in Strategy 2.13 the monodromy groups $H_{1}, H_{2}$ and $H_{\overline{2}}$ can be computed. All of them have index 75 in $\mathbb{B}_{3}$ and, using GAP4 [10], it can be seen that $H_{1}$ and $H_{2}$ are not conjugated. This implies that $H_{1}$ and $H_{\overline{2}}$ are not conjugated. Analogously, $H_{2}$, and $H_{\overline{2}}$ are not conjugated. Therefore, no homeomorphisms (resp. no oriented homeomorphism) exist between the associated pairs of fibered curves (and the line at infinity) for $s=s_{1}, s_{2}$ and $s=s_{1}, \bar{s}_{2}$ (resp. $s=s_{2}, \bar{s}_{2}$ ). 
TABLE 5 .

\begin{tabular}{|c|c|c|c|c|c|}
\hline$s=\sqrt{6}$ & $\sigma_{1}^{2} * \sigma_{2}$ & $\sigma_{2}^{5}$ & $\sigma_{2}^{2} * \sigma_{1}^{4}$ & $\left(\sigma_{2} \sigma_{1}^{-2}\right) * \sigma_{2}^{2}$ & $\sigma_{2} * \sigma_{1}^{6}$ \\
\hline$s=-\sqrt{6}$ & $\sigma_{1}^{2} * \sigma_{2}$ & $\sigma_{2}^{5}$ & $\sigma_{2}^{2} * \sigma_{1}^{4}$ & $\left(\sigma_{2} \sigma_{1}^{-2}\right) * \sigma_{2}^{2}$ & $\sigma_{1}^{6}$ \\
\hline
\end{tabular}

Example 4.5. The space $\mathbf{M}\left(\mathbb{E}_{6}, \mathbb{A}_{5}, \mathbb{A}_{4}, \mathbb{A}_{3}, \mathbb{A}_{1} ; 6\right)$ has exactly two points and representatives with equations in $\mathbb{Q}(\sqrt{6})[x, y, z]$ can be chosen as follows:

$$
\begin{aligned}
f_{s}(x, y, z):= & \left((-1940-792 s) x^{5}+(1940+792 s) x^{4} y+(3929+1604 s) z x^{4}\right. \\
+ & (-712 s-1744) z x^{3} y+(-832 s-2038) z^{2} x^{3} \\
+ & \left.(-84 s-206) z^{2} x^{2} y+(49+20 s) z^{3} x^{2}+(4 s+10) z^{3} y x+y^{2} z^{3}\right) \\
& \left(\left(-257-\frac{944}{9} s\right) x+y+\left(-\frac{569}{12}-\frac{349}{18} s\right) z\right),
\end{aligned}
$$

where $s^{2}=6$. Let us consider the triples associated with the $\mathbb{E}_{6}$-point $P=[0: 1: 0]$ and the tangent line $L=\{z=0\}$ at $P$. Braid monodromy representatives can be computed either using the program provided in [7] or applying the method of the real figure (see Table 55). We have followed the method (C) in Strategy 2.13. In this case, the monodromy groups $H_{\sqrt{6}}$ and $H_{-\sqrt{6}}$ have infinite index in $\mathbb{B}_{3}$. Considering the quotient of $\mathbb{B}_{3}$ by the normal subgroup generated by $\sigma_{1}^{60}$ and using GAP4 [10] one can find that the images of $H_{\sqrt{6}}$ and $H_{-\sqrt{6}}$ in the quotient group are not conjugated. The computation is not hard using GAP4 [10] since the index is 960 . We obtain a similar result as the one in Theorem 2 ,

\section{An APplication to Elliptic SURFACES}

Let $(\mathcal{C}, L, P)$ be a horizontal triple, where $\mathcal{C}$ is a sextic with rational singularities, and $P \in \mathcal{C}$ is a singular point of multiplicity 3. It is well known (see [15]) that a double covering $S$ of $\mathbb{P}^{2}$ ramified along $\mathcal{C}$ is a $K 3$-surface. Moreover (see again [15), the pencil of lines passing through $P$ gives $S$ an elliptic-surface-withsection structure $\pi_{P}: S \rightarrow \mathbb{P}^{1}$, where $\hat{L}$ is the preimage of $L$. In the sequel we will refer to this as the elliptic surface associated with $(\mathcal{C}, L, P)$. A braid monodromy of $(\mathcal{C}, L, P)$ can be lifted to a geometric monodromy of $\left(S, \pi_{P}, \hat{L}\right)$. This natural lifting is compatible with the equivalence relation defined for braid monodromy of curves and therefore the class of braid monodromies of $(\mathcal{C}, L, P)$ can be carried over to the geometric monodromies of $\left(S, \pi_{P}, \hat{L}\right)$. The purpose of this section is to develop this method and apply it to the previous example in order to obtain a result on elliptic fibrations.

The following is a general description of the homological monodromy of an elliptic surface. Suppose we fix an elliptic surface $\pi: S \rightarrow \mathbb{P}^{1}$ and a (not necessarily generic) fiber $F_{\infty}:=\pi^{-1}(\infty)$ on $S$. Let us denote by $\mathbb{C}$ the complement $\mathbb{P}^{1} \backslash\{\infty\}$. Consider $\mathscr{D}$ to be the branch locus of $\pi$. If we fix a base point $\star \in \mathbb{C} \backslash \mathscr{D}$ and denote its fiber $\pi^{-1}(\star)$ by $F$, then the fundamental group $\pi_{1}(\mathbb{C} \backslash \mathscr{D} ; \star)$ acts on $H_{1}(F):=H_{1}(F ; \mathbb{Z})$ producing a morphism $\nabla_{h}: \pi_{1}(\mathbb{C} \backslash \mathscr{D} ; \star) \rightarrow \operatorname{Aut}\left(H_{1}(F)\right)$.

We now have the following definition.

Definition 5.1. An $r$-tuple of matrices $\left(\tau_{1}, \ldots, \tau_{r}\right) \in S L(2, \mathbb{Z})^{r}$ represents a homological monodromy of $\left(S, \pi, F_{\infty}\right)$ if there exists $\star \in \mathbb{C} \backslash \mathscr{D}$ a base point, $\left\{x_{1}, \ldots, x_{r}\right\}$ a 
geometric basis of $\pi_{1}(\mathbb{C} \backslash \mathscr{D} ; \star)$, and $B:=\left\{\rho_{1}, \rho_{2}\right\}$ a basis of $H_{1}(F)$, such that the action $\nabla_{h}: \pi_{1}(\mathbb{C} \backslash \mathscr{D} ; \star) \rightarrow \operatorname{Aut}\left(H_{1}(F)\right)$ satisfies $\nabla_{h}\left(x_{i}\right)=\tau_{i}$, where the identification of $\operatorname{Aut}\left(H_{1}(F)\right)$ with $S L(2, \mathbb{Z})$ is given by means of the basis $B$.

Remark 5.2.

(1) The possible choices of a base point, a geometric basis of $\pi_{1}(\mathbb{C} \backslash \mathscr{D} ; \star)$ and a basis of $H_{1}(F)$ produce different $r$-tuples representing a homological monodromy of $\left(S, \pi, F_{\infty}\right)$. The class of such $r$-tuples defines an element in $\mathscr{M}_{r}(S L(2, \mathbb{Z}))$; see Definition 2.6. That is, two $r$-tuples represent a homological monodromy of $\left(S, \pi, F_{\infty}\right)$ if and only if both belong to the same class in $\mathscr{M}_{r}(S L(2, \mathbb{Z}))$.

(2) Note that, if $\left(\tau_{1}, \ldots, \tau_{r}\right) \in S L(2, \mathbb{Z})^{r}$ represents a homological monodromy of the triple $\left(S, \pi, F_{\infty}\right)$ and $F_{\infty}$ is a generic fiber, then the class of $\left(\tau_{1}, \ldots, \tau_{r}\right)$ does not depend on $F_{\infty}$. Such homological monodromy is known as the homological monodromy of the fibration $\pi: S \rightarrow \mathbb{P}^{1}$. In case $F_{\infty}$ is not generic, one can still recuperate the homological monodromy of the fibration $\pi: S \rightarrow \mathbb{P}^{1}$ from a representative $\left(\tau_{1}, \ldots, \tau_{r}\right) \in S L(2, \mathbb{Z})^{r}$ of a homological monodromy of $\left(S, \pi, F_{\infty}\right)$ as $\left(\tau_{1}, \ldots, \tau_{r}, \tau_{r+1}\right) \in S L(2, \mathbb{Z})^{r+1}$, where $\tau_{r+1}:=$ $\left(\tau_{r} \cdot \ldots \cdot \tau_{1}\right)^{-1}$.

(3) Note that, unless $F_{\infty}$ is a generic fiber, the equivalence relation among homological monodromies of triples is weaker than the equivalence relation of homological monodromies of fibered surfaces. Only if the local monodromy around $F_{\infty}$ is not conjugated to any other local monodromy around the special fibers of the fibered surface, then both concepts coincide. This is used in the proof of Theorem 5.4.

Let $(\mathcal{C}, L, P)$ be a horizontal triple as in the beginning of the section and $\left(S, \pi_{P}, \hat{L}\right)$ its associated elliptic fibration. Note that the branch locus of the vertical projection of $\mathcal{C}$ coincides with the branch locus $\mathscr{D}$ of the fibration. Moreover, after fixing a base point $\star \in \mathbb{C} \backslash \mathscr{D}$, the homological monodromy map $\nabla_{h}: \pi_{1}(\mathbb{C} \backslash \mathscr{D} ; \star) \rightarrow \operatorname{Aut}\left(H_{1}(\hat{L})\right)$ of the fibration factorizes through the monodromy map $\nabla: \pi_{1}(\mathbb{C} \backslash \mathscr{D} ; \star) \rightarrow \mathbb{B}_{3}$, that is, $\nabla_{h}=\psi \circ \nabla$. The map $\psi: \mathbb{B}_{3} \rightarrow S L(2, \mathbb{Z})$ can be defined as in (3.1) for appropriate choices of a basis in $H_{1}(\hat{L})$.

In other words, by Remark 5.2(1) and the previous discussion one has the following result.

Theorem 5.3. The homological monodromy of the elliptic fibration $\left(S, \pi_{P}, \hat{L}\right)$ is the $(\psi, S L(2, \mathbb{Z}))$-monodromy of $(\mathcal{C}, L, P)$; see Definition 2.10 .

Let us apply this construction to the example given in the previous section. Let $\mathcal{C}$ be a curve in the moduli space $\mathbf{M}:=\mathbf{M}\left(\mathbb{E}_{6}, \mathbb{A}_{7}, \mathbb{A}_{3}, \mathbb{A}_{2}, \mathbb{A}_{1} ; 6\right), P=\mathbb{E}_{6}$ and $\mathcal{H}_{P}$ the pencil through $P$. Blowing up $P$ one obtains a rational ruled surface fibered over $\mathbb{P}^{1}$ with a section $E$ and six singular fibers $L_{7}, L_{3}, L_{2}, L_{1}, L_{0}, L$ corresponding, respectively, to the singular points $\mathbb{A}_{7}, \mathbb{A}_{3}, \mathbb{A}_{2}, \mathbb{A}_{1}$, to a tangent line and to the line at infinity (see the beginning of $₫ 3$ ). The double covering ramified along $E \cup \tilde{\mathcal{C}}$ produces an elliptic $K 3$-surface (see Figure 5) of type $[1,2,3,4,6,8]$ according to the standard notation; see [14. This is an extremal elliptic $K 3$-surface listed as number 34 in Shimada and Zhang's Table; see [17.

One can state the following result concerning elliptic fibrations. 


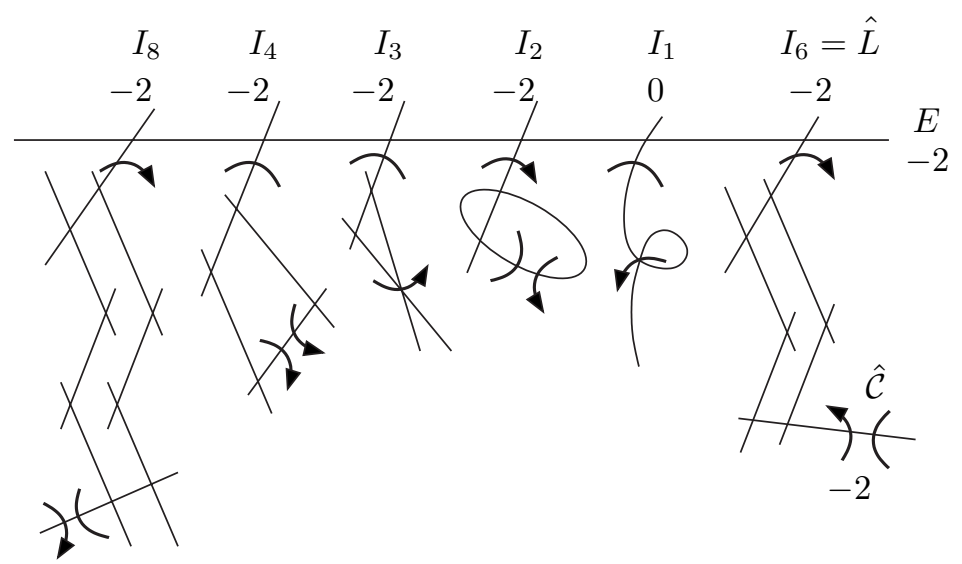

Figure 5. Surface $S$

Theorem 5.4. There are two elliptic fibrations of type $[1,2,3,4,6,8]$ with different homological monodromies.

Proof. Note that the triples $\left(\mathcal{C}_{\sqrt{2}}, L, P\right)$ and $\left(\mathcal{C}_{-\sqrt{2}}, L, P\right)$ constructed in $§ 3$ produce two elliptic fibrations $\left(S_{\sqrt{2}}, \pi_{\sqrt{2}}, \hat{L}\right)$ and $\left(S_{-\sqrt{2}}, \pi_{-\sqrt{2}}, \hat{L}\right)$. The calculations in the proof of Theorem 3.2 show that, if $\left(\tau_{1}^{+}, \ldots, \tau_{5}^{+}\right)$and $\left(\tau_{1}^{-}, \ldots, \tau_{5}^{-}\right)$are representatives of braid monodromies of the triples $\left(\mathcal{C}_{\sqrt{2}}, L, P\right)$ and $\left(\mathcal{C}_{-\sqrt{2}}, L, P\right)$, respectively, then $\left(\psi\left(\tau_{1}^{+}\right), \ldots, \psi\left(\tau_{5}^{+}\right)\right)$and $\left(\psi\left(\tau_{1}^{-}\right), \ldots, \psi\left(\tau_{5}^{-}\right)\right)$are not in the same orbit by the action of $\psi\left(\mathbb{B}_{5} \times \mathbb{B}_{3}\right)=\mathbb{B}_{5} \times S L(2, \mathbb{Z})$. In particular, the homological monodromies of the triples $\left(S_{\sqrt{2}}, \pi_{\sqrt{2}}, \hat{L}\right)$ and $\left(S_{-\sqrt{2}}, \pi_{-\sqrt{2}}, \hat{L}\right)$ are not equal. Now, by Remark $5.2(2)$, we know that $\tau_{+}:=\left(\tau_{1}^{+}, \ldots, \tau_{5}^{+},\left(\tau_{5}^{+} \cdot \ldots \cdot \tau_{1}^{+}\right)^{-1}\right)$ and $\tau_{-}:=\left(\tau_{1}^{-}, \ldots, \tau_{5}^{-},\left(\tau_{5}^{-} \cdot \ldots \cdot \tau_{1}^{-}\right)^{-1}\right)$ are representatives of the homological monodromies of the elliptic fibrations $\pi_{\sqrt{2}}$ : $S_{\sqrt{2}} \rightarrow \mathbb{P}^{1}$ and $\pi_{-\sqrt{2}}: S_{-\sqrt{2}} \rightarrow \mathbb{P}^{1}$, respectively. Suppose $\tau_{+}$and $\tau_{-}$are equivalent. Then there is a braid $\sigma \in \mathbb{B}_{6}$ and a matrix $M \in S L(2, \mathbb{Z})$ so that $\tau_{+}^{(\sigma, M)}=\tau_{-}$. The fiber $L$ is of type $I_{6}$ and there is no other fiber of this type in the fibrations. Note that the local homological monodromy of a fiber of type $I_{n}(n \in \mathbb{N})$ is $M_{n}:=\left(\begin{array}{ll}1 & n \\ 0 & 1\end{array}\right)$ and $M_{n}$ is not conjugate to $M_{m}$ in $S L(2, \mathbb{Z})$ unless $n=m$. Therefore, $\sigma$ has to fix the last coordinate of $\tau_{+}$and hence we can find $\sigma^{\prime} \in \mathbb{B}_{5}$ and $M^{\prime} \in S L(2, \mathbb{Z})$ satisfying $\left(\tau_{1}^{+}, \ldots, \tau_{5}^{+}\right)^{\left(\sigma^{\prime}, M^{\prime}\right)}=\left(\tau_{1}^{-}, \ldots, \tau_{5}^{-}\right)$which is a contradiction.

Remark 5.5. Compare the strength of this result with other descriptions of global homological braid monodromy of elliptic $K 3$-surfaces such as the one provided by M. Fukae in 9 .

\section{REFERENCES}

[1] H. Abelson, Topologically distinct conjugate varieties with finite fundamental group, Topology 13 (1974), 161-176. MR0349679 (50:2172)

[2] E. Artal, J. Carmona, and J.I. Cogolludo, On sextic curves with big Milnor number, Trends in Singularities (A. Libgober and M. Tibăr, eds.), Trends in Mathematics, Birkhäuser Verlag Basel/Switzerland, 2002, pp. 1-29. MR1900779 (2003d:14034)

[3] _ Braid monodromy and topology of plane curves, Duke Math. J. 118 (2003), no. 2, 261-278. MR1980995 (2004k:14015) 
[4] E. Artal, J. Carmona, J.I. Cogolludo, and H. Tokunaga, Sextics with singular points in special position, J. Knot Theory Ramifications 10 (2001), no. 4, 547-578. MR1831676 (2002c:14047)

[5] J. S. Birman, Braids, links, and mapping class groups, Princeton University Press, Princeton, N.J., 1974, Annals of Mathematics Studies, No. 82. MR0375281 (51:11477)

[6] E. Brieskorn, Automorphic sets and braids and singularities, Braids (Santa Cruz, CA, 1986), Amer. Math. Soc., Providence, RI, 1988, pp. 45-115. MR0975077 (90a:32024)

[7] J. Carmona, Monodromía de trenzas de curvas algebraicas planas, Ph.D. thesis, Universidad de Zaragoza, 2003.

[8] A.I. Degtyarëv, Isotopic classification of complex plane projective curves of degree 5, Leningrad Math. J. 1 (1990), no. 4, 881-904. MR.1027461 (91b:14041)

[9] M. Fukae, Monodromies of rational elliptic surfaces and extremal elliptic K3 surfaces, Preprint available at arXiv:math.AG/0205062.

[10] The GAP Group, Aachen, St Andrews, GAP - Groups, Algorithms, and Programming, Version 4.2, 2000, (http://www-gap.dcs.st-and.ac.uk/ gap).

[11] V. Kharlamov and V. Kulikov, Diffeomorphisms, isotopies, and braid monodromy factorizations of plane cuspidal curves, C. R. Acad. Sci. Paris Sér. I Math. 333 (2001), no. 9, 855-859. MR.1873224 (2003c:14047)

[12] A. Libgober, Invariants of plane algebraic curves via representations of the braid groups, Invent. Math. 95 (1989), no. 1, 25-30. MR0969412 (90a:14038)

[13] _ Characteristic varieties of algebraic curves, Applications of algebraic geometry to coding theory, physics and computation (Eilat, 2001), Kluwer Acad. Publ., Dordrecht, 2001, pp. 215-254. MR.1866902 (2003e:14008)

[14] R. Miranda and U. Persson, On extremal rational elliptic surfaces, Math. Z. 193 (1986), 537-558. MR0867347 (88a:14044)

[15] U. Persson, Double sextics and singular K-3 surfaces, Algebraic geometry, Sitges (Barcelona), 1983, Lecture Notes in Math., vol. 1124, Springer, Berlin, 1985, pp. 262-328. MR0805337 (87i:14036)

[16] J. P. Serre, Exemples de variétés projectives conjuguées non homéomorphes, C. R. Acad. Sci. Paris Sér. I Math. 258 (1964), 4194-4196. MR0166197 (29:3474)

[17] I. Shimada and D.-Q. Zhang, Classification of extremal elliptic K3 surfaces and fundamental groups of open K3 surfaces, Nagoya Math. J. 161 (2001), 23-54. MR.1820211 (2002d:14056)

[18] J.-G. Yang, Sextic curves with simple singularities, Tohoku Math. J. (2) 48 (1996), no. 2, 203-227. MR1387816 (98e:14026)

Departamento de Matemáticas, Campus Plaza de San Francisco s/n, E-50009 ZaraGOZA, SPAIN

E-mail address: artal@unizar.es

Departamento de Sistemas Informáticos y Programación, Universidad Complutense, Ciudad Universitaria S/n, E-28040 Madrid, Spain

E-mail address: jcarmona@sip.ucm.es

Departamento de Matemáticas, Campus Plaza de San Francisco s/n, E-50009 ZaraGOZA, SPAIN

E-mail address: jicogo@unizar.es 